\section{Intracranial tuberculoma associated with subdural hematoma}

Sir,

We report a 43-year-old man who presented with history of left hemicranial headache and right upper limb and facial focal onset motor seizures with secondary generalization of 3-month duration. There was no past history of tuberculosis, immunosuppressive states, diabetes mellitus or trauma. He was not from a low socioeconomic status and there was no family history of tuberculosis. On examination he had slurring of speech, decreased word output, bilateral papilloedema, right upper motor neuron facial paresis and right upper limb grade 4 muscle power. Computed tomogram (CT) of brain revealed a left posterior frontal ring-enhancing lesion with perilesional edema and an isodense frontoparietal subdural collection [Figure 1]. Magnetic resonance imaging (MRI) of brain revealed a left posterior frontal 2.8cm lobulated lesion, hypo to iso on T1W images, isointense to gray matter on $\mathrm{T} 2 \mathrm{~W}$ images with postgadolinium ring enhancement and perilesional oedema [Figure 2]. There was an associated frontoparietal subdural collection which was hyperintense on both T1W and T2W images suggestive of subacute blood. He underwent left frontotemporo-parietal craniotomy, evacuation of subdural hematoma and near total excision of the lesion. At surgery it was found that there was altered blood in the subdural space with thickened inner and outer membranes. The motor cortex was identified using electrophysiological monitoring and the lesion was just anterior to the motor cortex. It was well-defined with central necrotic material. The posterior wall of the lesion close to the motor cortex was left behind. The biopsy was reported as tuberculous granuloma. He was discharged on an 18-month course of antituberculous drugs. At 1-year follow-up he was asymptomatic and CT brain showed resolution of the lesion and hematoma [Figure 3].

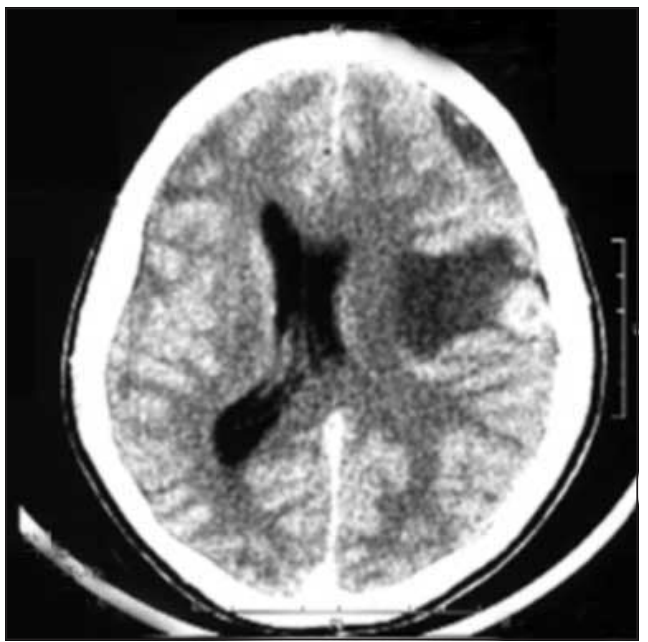

Figure 1: CT contrast image, axial section of brain showing a left posterior frontal ring enhancing lesion with perilesional edema associated with isodense left frontoparietal subdural collection.

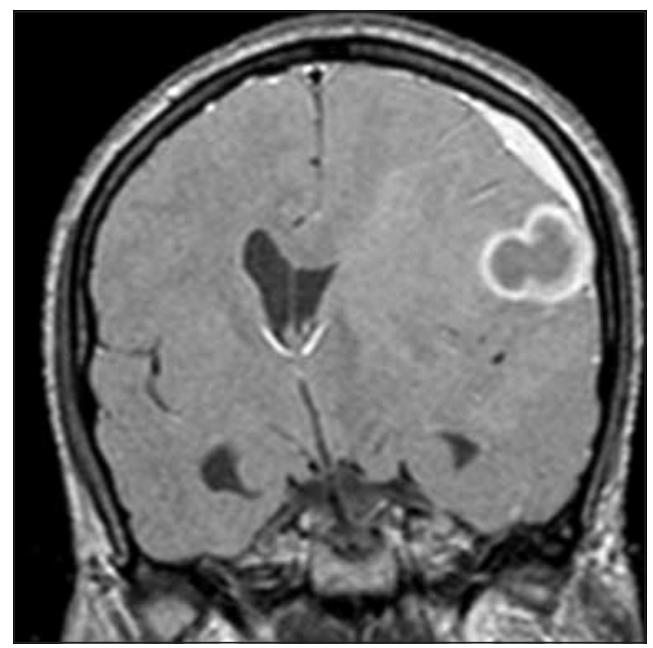

Figure 2: T1W coronal postgadolinium images showing a left frontal hypointense lesion with ring enhancement with mass effect and midline shift and an adjacent hyperintense subdural collection.

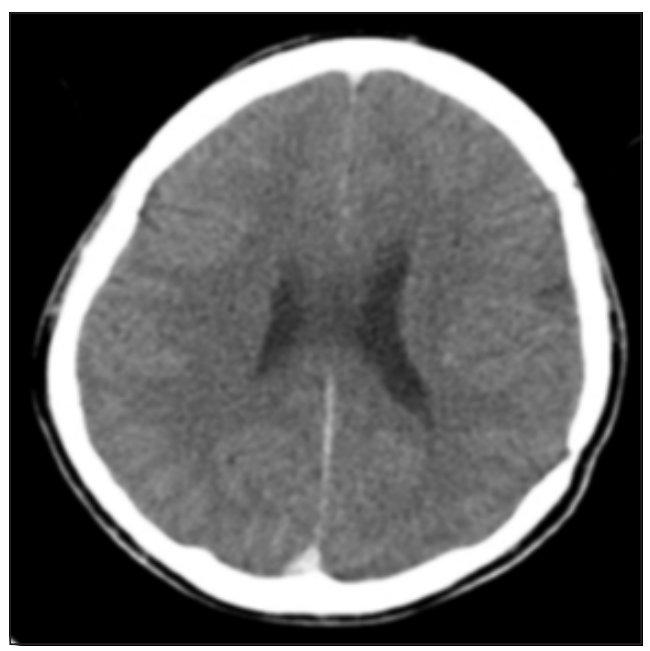

Figure 3: CT contrast image of brain at 1-year follow-up showing evidence of craniotomy with complete resolution of tuberculoma and hematoma 
Subdural hematomas in association with lesions like meningeal metastases, meningiomas and a cysticercus granuloma have been reported. ${ }^{[1-5]}$ Recently there has been a report of cerebellar hematoma resulting from a tuberculoma ${ }_{-}^{[6]}$ Metastases are the most common lesions known to be associated with subdural hematomas. ${ }^{[3]}$ The pathogenesis of subdural hematoma in these cases is probably due to circulatory disturbance in the duramater caused by invasion of dural veins or capillaries by tumor or tumor cells emboli. This could result in bleeding from the capillaries of the inner vascular layer of the dura and formation of subdural hematoma. In other cases, where the metastasis was a manifestation of diffuse end-stage disease, subdural hematoma was a sequalae to disseminated intravascular coagulopathy. ${ }^{[2]}$

Tumors with a propensity to bleed, such as meningiomas, if located superficially could bleed severely or have a hemorrhagic effusion into the subdural space and present with a subdural hematoma. ${ }^{[3,4]}$ The proposed mechanisms for subdural hematomas associated with meningioma are fragile thin-walled vessels, direct invasion by the tumor and long-standing ischemia producing hyalinization of the tissue, thereby changing the hemodynamics and producing vascular stress in thin-walled vessels leading to rupture. In the case of cerebellar hematoma, presence of phlebitis provides a histological evidence for the probable pathophysiology. ${ }^{[6]}$ We have previously reported a patient with cysticercus granuloma who had an associated subdural effusion. ${ }^{[5]}$ In that report it is presumed that the inflammation around the cysticercus granuloma, having spread to the adjacent meninges and bridging veins in the subdural space led to the formation of a transudate. Although these cases support the possibility of thrombophlebitis of the draining cortical veins, in the absence of definitive histological features of phlebitis in our case, the subdural hematoma and tuberculoma could be just coincidental findings.

Sujit Kumar G Samson, Santosh Isaac Poonoose, Vedantam Rajshekhar Department of Neurological Sciences, Christian Medical College, Vellore, Tamilnadu, India

Address for correspondence: Dr. Vedantam Rajshekhar, Department of Neurological Sciences, Christian Medical College, Vellore, Tamil Nadu-632 004, India. E-mail: rajshekhar@cmcvellore.ac.in

\section{References}

1. N'dri Oka D, Varlet G, Boni N, Braolet E, Boukassa L, Ba Zeze V. Dural metastases of prostatic adenocarcinoma presenting as acute intracranial subdural haematoma. J Neuroradiol 2000;27:282-4.

2. Hirashima Y, Kamiyama K, Endo S, Takaku A. Subdural haematoma due to metastatic dural carcinomatosis associated with DIC. No Shinkei Geka 1983;11:651-6.
3. Chaskis C, Raftopoulos C, Noterman J, Flament Durand J, Brotchi J. Meningioma associated with subdural haematoma: A report of two cases and review of literature. Clin Neurol Neurosurg 1992;94:269-74.

4. Okuno S, Touho H, Ohnishi H, Karasawa J. Falx meningioma presenting as acute subdural haematoma: Case report. Surg Neurol 1999;52:180-4.

5. Rajshekhar V. Cerebral cysticercus granuloma associated with a subdural effusion. Neruol India 2001;49:182-4.

6. Ranjan M, Saritha A, Mahadevan A, Shankar SK, Sampath S. Cerebellar tuberculoma presenting as hematoma- a case report and pathophysiological consideration. Br J Neurosurg 2009;23:203-5.

\begin{tabular}{|l|l|}
\hline \multicolumn{2}{|c|}{ Access this article online } \\
\hline Quick Response Code: & Website: \\
\hline & www.ruralneuropractice.com \\
\cline { 2 - 2 } & \\
\hline & \\
\hline
\end{tabular}

